\title{
BRAIN-COMPUTER INTERFACES FOR 1-D AND 2-D CURSOR CONTROL: DESIGNS USING VOLITIONAL CONTROL OF THE EEG SPECTRUM OR STEADY-STATE VISUAL EVOKED POTENTIALS
}

\author{
Leonard J. Trejo, NASA Ames Research Center, Moffett Field, CA, USA \\ Bryan Matthews, QSS Group, Inc., Lanham, MD, USA \\ Roman Rosipal, Austrian Research Institute for Artificial Intelligence, Vienna, Austria
}

We have developed and tested two EEG-based brain-computer interfaces (BCI) for users to control a cursor on a computer display. Our system uses an adaptive algorithm, based on kernel partial least squares classification (KPLS), to associate patterns in multichannel EEG frequency spectra with cursor controls. Our first BCI, Target Practice, is a system for one-dimensional device control, in which participants use biofeedback to learn voluntary control of their EEG spectra. Target Practice uses a KPLS classifier to map power spectra of 30-electrode EEG signals to rightward or leftward position of a moving cursor on a computer display. Three subjects learned to control motion of a cursor on a video display in multiple blocks of 60 trials over periods of up to six weeks. The best subject's average skill in correct selection of the cursor direction grew from $58 \%$ to $88 \%$ after 13 training sessions. Target Practice also implements online control of two artifact sources: a) removal of ocular artifact by linear subtraction of wavelet-smoothed vertical and horizontal EOG signals, b) control of muscle artifact by inhibition of BCI training during periods of relatively high power in the 40-64 Hz band.

The second BCI, Think Pointer, is a system for two-dimensional cursor control. Steady-state visual evoked potentials (SSVEP) are triggered by four flickering checkerboard stimuli located in narrow strips at each edge of the display. The user attends to one of the four beacons to initiate motion in the desired direction. The SSVEP signals are recorded from eight electrodes located over the occipital region. A KPLS classifier is individually calibrated to map multichannel frequency bands of the SSVEP signals to right-left or up-down motion of a cursor on a computer display. The display stops moving when the user attends to a central fixation point. As for Target Practice, Think Pointer also implements wavelet-based online removal of ocular artifact; however, in Think Pointer muscle artifact is controlled via adaptive normalization of the SSVEP. Training of the classifier requires about three minutes. We have tested our system in real-time operation in three human subjects. Across subjects and sessions, control accuracy ranged from $80 \%$ to $100 \%$ correct with lags of 1-5 seconds for movement initiation and turning. We have also developed a realistic demonstration of our system for control of a moving map display (http://ti.arc.nasa.gov/story.php?id=265\&sec=4).

Supported by the Human-Centered Computing Project of the NASA Intelligent Systems Program, and the Information Technology Strategic Research Project of the NASA Computing, Information and Communications Technology Program. 